\title{
Current status of projects for developing cancer-related clinical practice guidelines in Japan and recommendations for the future
}

\author{
Masafumi Imamura ${ }^{1}$ Koichi Hirata ${ }^{1,16} \cdot$ Michiaki Unno $^{2} \cdot$ Kinj Kamiya $^{3} \cdot$ Mitsukazu Gotoh $^{4} \cdot$ Hiroyuki Konno $^{5}$. \\ Akiko Shibata $^{6} \cdot$ Kenichi Sugihara $^{7} \cdot$ Arata Takahashi $^{8} \cdot$ Masahiko Nishiyama $^{9} \cdot$ Kenichi Hakamada $^{10}$. \\ Tsuguya Fukui ${ }^{11} \cdot$ Toshiharu Furukawa $^{12} \cdot$ Tsunekazu Mizushima $^{13} \cdot$ Masamichi Mizuma $^{2} \cdot$ Hiroaki Miyata $^{14}$. \\ Masaki Mori ${ }^{13} \cdot$ Ichiro Takemasa $^{1} \cdot$ Toru Mizuguchi $^{1} \cdot$ Toshiyoshi Fujiwara $^{15}$
}

Received: 2 April 2018 / Accepted: 17 August 2018 / Published online: 24 August 2018

(c) The Author(s) 2018

\begin{abstract}
Background The current status and adoption of cancer-related clinical practice guidelines in Japan has not been elucidated yet. The purpose of this study was to propose roles and suggestions to develop future cancer-related clinical guidelines. Methods A questionnaire consisting of four domains with a total of 17 questions was developed. We distributed the questionnaire to 28 specific academic organizations in Japan which have developed any cancer-related clinical practice guidelines and which were funded by the Ministry of Health, Labor, and Welfare.

Results Most organizations have investigated nationwide dissemination and adoption of clinical practice guidelines. The rate of adoption in clinical practice was estimated at approximately $\geq 70 \%$. However, organizations with smaller budgets reported surveying approximately $60 \%$ of the time, whereas the ones with larger budgets reported approximately $100 \%$ success in surveying about their guidelines. The presidents of the organizations agreed that a new organization operated directly by the national government was necessary.

Conclusion In Japan, to develop cancer-related clinical practice guidelines, a study of clinical validation is necessary. Sufficient funds must be available to support the project to maintain and revise the guidelines. Furthermore, legal and ethical issues should be solved before establishing any registry system.
\end{abstract}

Keywords Cancers $\cdot$ Clinical practice guidelines $\cdot$ Plan-do-check-act cycle $\cdot$ Japan Society of Clinical Oncology

\section{Introduction}

Many clinical practice guidelines are developed worldwide in various fields including medicine, oncology, and surgery. The essential purpose of creating cancer-related clinical practice guidelines is to improve public knowledge and understanding for improving the quality of cancer treatment [1-3]. To date, we have focused on promoting medical recommendations and clinical evidence in the guidelines. In addition, we have also worked to validate their effectiveness after publishing them in clinical fields. However, most levels of clinical evidence were not high enough to support clinical

Masafumi Imamura

imamura@sapmed.ac.jp

Koichi Hirata

kouichi.hirata@jrhokkaido.co.jp

Extended author information available on the last page of the article practice guidelines and their recommendations. Therefore, additional clinical studies are needed to provide more reliable and validated medical evidence.

Cancer-related clinical practice guidelines are becoming popular in the public despite the transition of the medical environment in Japan. In this situation, the guidelines should be objective and sequential for reflecting a high medical consciousness in the public. Therefore, we are finding more situations where we need to establish a system to develop new guidelines while maintaining the existing clinical practice guidelines [4].

Therefore, we attempted to clarify the coordination among the existing organizations that disseminate cancerrelated clinical practice guidelines in Japan. We also proposed investigating what types of problems organizations might be encountering under the current circumstances. We developed a questionnaire for academic organizations in Japan about experiences of each organization regarding 
the adoption of cancer-related clinical practice guidelines in Japan, the academic services which each organization provides to evaluate the clinical impact and adoption of the guidelines, and the current status of recent clinical research. The questionnaire consisted of 17 questions associated with clinical practice guidelines, six questions about conflicts of interest, 15 questions about cancer registries, seven questions about clinical research/ analysis, six questions related to medical information ethics, and four questions related to funding. In this study, we focused on the specific domain of the questionnaire regarding clinical practice guidelines for the treatment of cancer.

The purpose of this study was to clarify the current status of each academic organization in developing clinical practice guidelines for the treatment of cancer in Japan, and to propose recommendations for the development of clinical guidelines in the future.

\section{Subjects and methods}

\section{Subjects}

A search using two key terms, "cancer" and "clinical practice guidelines," was conducted in the databases of the National Cancer Center Japan, the Japan Society of Clinical Oncology, and the Medical Information Network Distribution Service (Minds) in November 2016 to identify academic organizations which were responsible for publishing clinical practice guidelines dealing with "cancer." We identified 37 guidelines in 28 organizations (Table 1). We conducted a survey of 28 presidents or chairpersons from the organizations which develop cancer-related clinical practice guidelines using a questionnaire.

We analyzed the status of cancer-related clinical practice guidelines in Japan and identified the current situation and problems. Based on our findings, we developed recommendations regarding the creation of cancer-related clinical practice guidelines in the future.
Table 1 List of academic organizations in Japan which published cancer-related clinical practice guidelines

\begin{tabular}{|c|c|}
\hline \# & Academic organizations \\
\hline 1 & The Research Group for Rare Neoplasms of Japan \\
\hline 2 & Japanese Society for Cancer of the Colon and Rectum \\
\hline 3 & Japanese Gastric Cancer Association \\
\hline 4 & The Japan Society of Hepatology \\
\hline 5 & Japanese Society of Hepato-Biliary-Pancreatic Surgery \\
\hline 6 & Japan Society of Clinical Oncology: Proper management using Antiemetic agent \\
\hline 7 & Japan Society of Clinical Oncology: Proper management using G-CSF \\
\hline 8 & Japanese Society for Palliative Medicine \\
\hline 9 & Japanese Society of Hematology \\
\hline 10 & Japanese Society of Oral Oncology \\
\hline 11 & Japanese Society of Thyroid Surgery \\
\hline 12 & The Japanese Society of Pediatric Hematology Oncology \\
\hline 13 & The Japan Esophageal Society \\
\hline 14 & Japan Neuroendocrine Tumor Society \\
\hline 15 & Japan Pancreas Society \\
\hline 16 & The Japanese Orthopedic Association \\
\hline 17 & Japan Society for Head and Neck Cancer \\
\hline 18 & Japanese Breast Cancer Society \\
\hline 19 & Japan Association of Breast Cancer Screening \\
\hline 20 & The Japan Society for Neuro-Oncology \\
\hline 21 & The Japan Lung Cancer Society \\
\hline 22 & The Japanese Urological Association \\
\hline 23 & Japanese Skin Cancer Society \\
\hline 24 & Japan Society of Gynecologic Oncology \\
\hline 25 & Japanese Society for Radiation Oncology \\
\hline 26 & The Japanese Association of Rehabilitation Medicine \\
\hline 27 & Japanese Society of Medical Oncology \\
\hline 28 & The Japanese Lymphedema Society \\
\hline
\end{tabular}




\section{Questionnaire}

The following questionnaire was developed and implemented from October to November 2016. The questionnaire consisted of 19 questions regarding the following: (1) awareness of the national popularization; (2) clinical values of the guidelines; (3) miscellaneous situations and funds (Table 2). The questionnaire was distributed by a study group of the Ministry of Health, Labor, and Welfare (2015-Cancer countermeasure, General-003).

\section{Results}

All 28 academic organizations responsible for publishing clinical practice guidelines on treating cancers responded to our questionnaire (100\% response rate).

\section{Awareness of the national popularization}

Half of the academic organizations $(N=14 / 28)$ had information about the nationwide adoption of their clinical practice guidelines (Table 3: Question 1), with 79\% of organizations $(N=11 / 14)$ reporting that the nationwide adoption rate of their guidelines was $41 \%$ or more (Table 3: Question 1-a). Among those, $73 \%$ of organizations $(N=8 / 11)$ believed that the adoption rate of their clinical guidelines was $71 \%$ or more.

\section{Clinical values of the guidelines}

In this survey (Table 4: Question 2), 68\% of organizations $(N=19 / 28)$ claimed that they had already investigated the clinical values of recommendations for medical practice which were presented in their clinical guidelines. For the nine organizations that did not conduct such an investigation, the main reasons were the lack of a registry and/or analytical systems and insufficient funds (Table 4: Question 2-a).

Table 3 Awareness of the national popularization

Q1: Have you ever surveyed the national popularization of clinical guidelines on cancers in your field $(N=28)$ ?

Yes, we have $(N=14 ; 50 \%)$

No, we have not yet $(N=14 ; 50 \%)$

Q1-a: If yes, what rate of national popularization of the guidelines did you achieve in the survey $(N=14)$ ?

\begin{tabular}{llc}
\hline Estimated rate (\%) & $N$ & $\%$ \\
\hline $41-50$ & 1 & 7 \\
$51-60$ & 1 & 7 \\
$61-70$ & 1 & 7 \\
$71-80$ & 3 & 22 \\
$81-90$ & 3 & 22 \\
$91-100$ & 2 & 14 \\
No reply & 3 & 22 \\
\hline
\end{tabular}

Table 2 Contents of questionnaire survey pertaining to the development and publication of cancer-related clinical practice guidelines

1 Have you ever surveyed the national popularization of clinical guidelines on cancers in your field?

1-a If yes, what rate of national popularization of the guidelines did you achieve in the survey?

2 Have you ever investigated clinical values of "The recommendations for medical practice," which you have presented in the guidelines?

2-a If no, what do you think the obstacle was?

2-b If yes, how many clinical guidelines have you investigated?

2-c If you publish a paper about the clinical values of any recommendations for medical practice, please list them in terms of the published year

2-d How did you register the cases that met the inclusion criteria of the research?

3 Did you have any ideas regarding the current situation where the governance and management of clinical practice guidelines is the responsibility of academic organizations?

3-a Have you come up with any idea regarding current situations of establishing clinical guidelines on cancers in Japan?

3-b What kind of organization do you recommend to manage making guidelines?

3-c What kind of funds do you recommend to operating new organization?

3-d What is your annual budget to operate the guidelines, including making and presenting?

3-e Relations between the rate of surveillance investigating popularization and annual budget on clinical practice?

3-f What is essential to overcome difficult situations while conducting medical quality assessment?

3-g What are your thoughts on the current situation wherein the academic organization is responsible for the total management of establishing cancer-related clinical guidelines?

4 Do you publish information for patients about clinical guidelines or present that information on the internet?

4-a What was your intention behind publishing clinical guidelines for the patients?

4-b What were the difficulties faced during process optimization?

4-c When do you plan to revise them? 
Table 4 Clinical values of the guidelines

Q2: Have you ever investigated clinical values of "The recommendations for medical practice," which you have presented in the guidelines $(N=28)$ ?

Yes, we have $(N=19 ; 68 \%)$

No, we have not yet $(N=9 ; 32 \%)$

Q2-a: If no, what do you think the obstacle was $(N=9)$ ?

\begin{tabular}{lll}
\hline Obstacles & $N$ & $\%$ \\
\hline Lack of registry and/or analytical systems & 9 & 100 \\
Short funding & 9 & 100 \\
Regal problem & 3 & 33 \\
Ethical problem & 3 & 33 \\
Others & 2 & 22 \\
\hline
\end{tabular}

Table 5 Miscellaneous situations and funds

Q3-a: Have you come up with any idea regarding current situations of establishing clinical guidelines on cancers in Japan $(N=28)$ ?

\begin{tabular}{lll}
\hline Opinions & $N$ & $\%$ \\
\hline We are satisfied with the current systems & 8 & 29 \\
Creating a new supervisory organization is necessary & 19 & 68 \\
Others & 1 & 3 \\
\hline
\end{tabular}

Q3-b: What kind of organization do you recommend to manage making guidelines $(N=28)$ ?

\begin{tabular}{lll}
\hline Opinions & $N$ & $\%$ \\
\hline New organization directly under control by the government & 4 & 21 \\
Existing academic organizations with coordination & 11 & 58 \\
A private organization & 3 & 16 \\
Others & 1 & 5 \\
\hline
\end{tabular}

Q3-c: What kind of funds do you recommend to operating new organization $(N=19)$ ?

\begin{tabular}{lll}
\hline Opinions & $N$ & $\%$ \\
\hline Funds from the national budget & 16 & 84 \\
Funds from academic organizations themselves & 3 & 16 \\
\hline
\end{tabular}

Q3-d: What is your annual budget to operate the guidelines, including making and presenting $(N=28)$ ?

\begin{tabular}{|c|c|c|c|c|}
\hline \multicolumn{2}{|l|}{ Annual amount } & $\mathrm{N}$ & \multicolumn{2}{|l|}{$\%$} \\
\hline \multicolumn{2}{|l|}{ Less than 1 million yen } & 8 & \multicolumn{2}{|l|}{29} \\
\hline \multicolumn{2}{|l|}{ One million yen to less than 3 million yen } & 13 & \multicolumn{2}{|l|}{46} \\
\hline \multicolumn{2}{|l|}{ Three million yen and more } & 4 & \multicolumn{2}{|l|}{14} \\
\hline \multicolumn{2}{|l|}{ No reply } & 3 & \multicolumn{2}{|l|}{11} \\
\hline \multicolumn{5}{|c|}{ Q3-e: Relations between the rate of surveillance investigating popularization and annual budget on clinical practice guidelines $(N=25)$ ? } \\
\hline \multirow[t]{2}{*}{ Annual budget } & \multicolumn{2}{|c|}{ Investigated or under investigation } & \multicolumn{2}{|c|}{ No further investigation } \\
\hline & $N$ & $\%$ & $N$ & $\%$ \\
\hline Less than 1 million yen & 5 & 62 & 3 & 38 \\
\hline One million yen to less than 3 million yen & 8 & 62 & 5 & 38 \\
\hline Three million yen and more & 4 & 100 & 0 & 0 \\
\hline
\end{tabular}


In terms of the funding needed to disseminate and manage clinical guidelines in each organization, $46 \%$ of respondents $(N=13 / 28)$ reported annual costs in the range of 1-3 million yen. On the other hand, $29 \%$ of organizations $(N=8 / 28)$ were spending less than 1 million yen (Table 5: Question 3-d).

The relationship between the rate of surveillance to investigate the adoption and the annual budget for clinical practice guidelines was shown in Table 5: Question 3-e. All academic organizations who reported annual operating costs of 3 million yen or more had already investigated the adoption of their clinical practice guidelines. On the other hand, organizations with a budget of less than 3 million yen had an investigation rate of around $60 \%$.

\section{Discussion}

Various cancer-related clinical practice guidelines have been developed and disseminated by academic organizations in Japan to improve the quality of oncological care. The academic organizations also play a role in educating medical personnel on recommended clinical practices. Educational systems to communicate the recommendations found in clinical practice guidelines to those in the medical field have recently become popular. However, an adequate system for updating and revising the clinical practice guidelines after launching the initial guidelines does not exist. Therefore, we need to identify the circumstances currently facing academic organizations and what needs to be done to address areas of concern.

Working as a research team of the Ministry of Health, Labor, and Welfare, we prepared a questionnaire regarding the adoption of cancer-related clinical practice guidelines in Japan, the academic services in each specific organization to evaluate the clinical impact and adoption of the guidelines, and the status of recent clinical research. Our aims were to determine the current status of the development of cancerrelated clinical practice guidelines in Japan and to develop recommendations for the future.

It is necessary to investigate the national popularization of clinical practice guidelines after they are disseminated. It is also important to investigate whether medical recommendations presented in the guidelines are being followed, and to verify the outcomes in clinical practice. These processes are a plan-do-check-act cycle of continual improvement and help to establish the essential systems to revise guidelines and to continually improve the quality of oncological management of patients [5].

Based on the results of this questionnaire, organizations that conducted surveys on the nationwide adoption of their clinical guidelines found relatively high rates of adoption. However, approximately $60 \%$ of organizations had insufficient annual funding for the management of their clinical practice guidelines. Approximately $30 \%$ of organizations did not conduct any evaluation to determine the extent to which their recommended medical practices were being implemented. The establishment of a registration and analysis system and securing adequate funding were cited as possible solutions to these problems and will be challenges for the future.

Regarding the management of cancer-related clinical practice guidelines, it was confirmed that many organizations agree with the need to create a new nationwide supervisory organization. The preferred structure was to have a national government commission as a third-party supervisory organization. Most respondents felt that a representative, existing, cross-sectional academic organization would be more appropriate than an organization under the direct control of the Ministry of Health, Labor, and Welfare. Specifically, the Japan Society of Clinical Oncology, which has long led a comprehensive effort aimed at the dissemination and development of new Japanese cancer-related clinical practice guidelines, was named as a candidate. A consortium of organizations that contribute to the fields of hematological neoplasm or drug therapy, including the Japanese Society of Medical Oncology, is also a potential candidate.

However, it also may be possible to directly operate a wide-ranging supervisory body at the nationwide level, as is done in the US. In this case, structure and regulations that ensure freedom in the utilization system become essential [6]. An earlier survey [7] found that the Japan Society of Clinical Oncology was again considered the most appropriate third-party organization for this role. Going forward, we hope to proceed with investigations into an organization that will play a central role in the development of cancerrelated clinical practice guidelines. Two important issues that remain after the analysis of survey responses include the following: (1) substantial differences seen among the academic organizations with respect to trends in the business situation regarding the dissemination/evaluation of cancerrelated clinical practice guidelines and (2) accessibility of data for additional clinical studies to provide more reliable and validated medical evidence. To solve these problems, we are continuing our efforts as a study group, and we are aspiring to achieve concrete improvements. Sufficient funds must be available to support the project to maintain and revise the guidelines. Furthermore, legal and ethical issues should be solved before establishing any registry system.

\section{Conclusion}

Cancer-related clinical practice guidelines in Japan have increased in popularity over time. However, investigations of the rates of adoption and implementation of the clinical recommendations in the guidelines and the impact and clinical outcomes after the publication of clinical guidelines were still lower than acceptable. The reasons for this situation 
were inadequate budget and a lack of systems for registry and analysis. The important issues that were revealed in this study include large gaps among academic organizations regarding adoption and the assessment of the practice guidelines. Furthermore, a new organization in Japan, funded by the government, is needed to support the management of the current cancer-related clinical practice guidelines and the development of additional guidelines.

Acknowledgements This project was made possible by the following Health and Labor Sciences Research Grant: "Promotion of large-scale cohort studies based on organ cancer registration in cooperation with national cancer registration and study of construction of an National Clinical Database long-term prognostic input system for a high-quality medical database" (Principal Investigator: Koichi Hirata, 2015-2017: $\mathrm{H} 27-003)$. We are grateful to all the respondents who belong to academic societies developing cancer-related clinical practice guidelines. The 28 societies that responded to our questionnaire were The Research Group for Rare Neoplasms of Japan, Japanese Society for Cancer of the Colon and Rectum, Japanese Gastric Cancer Association, The Japan Society of Hepatology, Japanese Society of Hepato-Biliary-Pancreatic Surgery, Japan Society of Clinical Oncology: Proper management using Antiemetic agent, Japan Society of Clinical Oncology: Proper management using G-CSF, Japanese Society for Palliative Medicine, Japanese Society of Hematology, Japanese Society of Oral Oncology, Japanese Society of Thyroid Surgery, The Japanese Society of Pediatric Hematology Oncology, The Japan Esophageal Society, Japan Neuroendocrine Tumor Society, Japan Pancreas Society, The Japanese Orthopedic Association, Japan Society for Head and Neck Cancer, Japanese Breast Cancer Society, Japan Association of Breast Cancer Screening, The Japan Society for Neuro-Oncology, The Japan Lung Cancer Society, The Japanese Urological Association, Japanese Skin Cancer Society, Japan Society of Gynecologic Oncology, Japanese Society for Radiation Oncology, The Japanese Association of Rehabilitation Medicine, Japanese Society of Medical Oncology, and The Japanese Lymphedema Society.

\section{Compliance with ethical standards}

Conflict of interest The authors have no conflicts of interest to declare.

Open Access This article is distributed under the terms of the Creative Commons Attribution 4.0 International License (http://creativeco mmons.org/licenses/by/4.0/), which permits unrestricted use, distribution, and reproduction in any medium, provided you give appropriate credit to the original author(s) and the source, provide a link to the Creative Commons license, and indicate if changes were made.

\section{References}

1. Field MJ, Lohr KN (1990) Clinical practice guidelines: directions for a new program. National Academies Press, Washington

2. Grimshaw JM, Russell IT (1993) Effect of clinical guidelines on medical practice: a systematic review of rigorous evaluations. Lancet 342:1317-1322

3. Worrall G, Chaulk P, Freake D (1997) The effects of clinical practice guidelines on patient outcomes in primary care: a systematic review. Can Med Assoc J 156:1705-1712

4. Shimbo T, Fukui T, Ishioka C et al (2010) Quality of guideline development assessed by the evaluation committee of the Japan society of clinical oncology. Int J Clin Oncol 15:227-233

5. Ansari S, Rashidian A (2012) Guidelines for guidelines: are they up to the task? A comparative assessment of clinical practice guideline development handbooks. PLoS One 7:e49864

6. Winn RJ (2000) The NCCN guidelines development process and infrastructure. Oncology 14:26-30

7. Furuhata T, Hirata K, Wakao F et al (2014) Questionnaire survey for the development and publication of cancer clinical practice guidelines in Japan. Int J Clin Oncol 19:771-778

\section{Affiliations}

\section{Masafumi Imamura ${ }^{1} \cdot$ Koichi Hirata $^{1,16} \cdot$ Michiaki Unno $^{2} \cdot$ Kinj Kamiya $^{3} \cdot$ Mitsukazu Gotoh $^{4} \cdot$ Hiroyuki Konno ${ }^{5} \cdot$ Akiko Shibata $^{6} \cdot$ Kenichi Sugihara $^{7} \cdot$ Arata Takahashi $^{8} \cdot$ Masahiko Nishiyama $^{9} \cdot$ Kenichi Hakamada $^{10}$. Tsuguya Fukui $^{11} \cdot$ Toshiharu Furukawa $^{12} \cdot$ Tsunekazu Mizushima $^{13} \cdot$ Masamichi Mizuma $^{2} \cdot$ Hiroaki Miyata $^{14}$. Masaki Mori $^{13} \cdot$ Ichiro Takemasa $^{1} \cdot$ Toru Mizuguchi $^{1} \cdot$ Toshiyoshi Fujiwara $^{15}$}

1 Department of Surgery, Surgical Oncology and Science, Sapporo Medical University, South 1, West 16, Chuo-ku, Sapporo 060-8543, Japan

2 Department of Surgery, Tohoku University Graduate School of Medicine, Sendai, Japan

3 Second Department of Surgery, Hamamatsu University School of Medicine, Hamamatsu, Japan

4 Osaka General Medical Center, Osaka, Japan

5 Hamamatsu University School of Medicine, Hamamatsu, Japan

6 National Cancer Center, Center for Cancer Control and Information Services, Tokyo, Japan

7 Tokyo Medical and Dental University, Tokyo, Japan
8 Department of Health Policy and Management, School of Medicine, Keio University, Tokyo, Japan

9 Department of Molecular Pharmacology and Oncology, Gunma University Graduate School of Medicine, Maebash, Japan

10 Department of Gastroenterological Surgery, Hirosaki University Graduate School of Medicine, Hirosaki, Japan

11 St. Luke's International University, Tokyo, Japan

12 Keio University Law School, Tokyo, Japan

13 Department of Gastroenterological Surgery, Graduate School of Medicine, Osaka University, Osaka, Japan

14 The University of Tokyo, Healthcare Quality Assessment, Tokyo, Japan 
15 Department of Gastroenterological Surgery, Okayama University Graduate School of Medicine, Dentistry and Pharmaceutical Sciences, Okayama, Japan
16 JR Sapporo Hospital, North 3, East 1, Chuo-ku, Sapporo 060-0033, Japan 